\title{
Polarimetry in Planetary Science-A Step Forward with the VLT and a Need for the ELTs
}

\author{
H. Boehnhardt $\cdot$ G. P. Tozzi $\cdot$ M. Sterzik $\cdot$ S. Bagnulo $\cdot$ \\ L. Kolokolova $\cdot$ K. Muinonen
}

Received: 31 January 2009/Accepted: 26 May 2009/Published online: 19 June 2009

(C) The Author(s) 2009. This article is published with open access at Springerlink.com

\begin{abstract}
We present a brief review of polarimetric measurements of solar system objects, both linear and circular, obtained with the FORS1 instrument at the Very Large Telescope VLT over the past years. A number of first and new results have been obtained by using this unique observing mode at an $8 \mathrm{~m}$ class telescope, among them polarimetry of faint planetary bodies like near-Earth asteroids, Kuiper Belt objects and cometary nuclei, spectropolarimetry of cometary coma material and of the Earthshine of the Moon (in order to verify that life exists on Earth!). We outline the science cases for planetary polarimetry at a future Extremely Large Telescope ELT and provide high level requirements for polarimetric equipment to be used at the ELTs for the study of the science cases described.
\end{abstract}

Keywords Polarimetry - Near-Earth asteroids · Kuiper Belt objects ·

Cometary nucleus $\cdot$ Coma material $\cdot$ Life science $\cdot$ ELT

H. Boehnhardt (四)

MPI for Solar System Research, Katlenburg-Lindau, Germany

e-mail: hboehnha@linmpi.mpg.de

G. P. Tozzi

INAF Arcetri Observatory, Florence, Italy

M. Sterzik

European Southern Observatory, Santiago de Chile, Chile

S. Bagnulo

Armagh Observatory, Armagh, UK

L. Kolokolova

University of Maryland, College Park, MD, USA

K. Muinonen

University of Helsinki, Helsinki, Finland 


\section{Stokes Parameters and the FORS1 Instrument Used for Solar System Object Polarimetry}

Polarimetry is still considered a bit of an esoteric tool for exploring the bodies in the solar system. It is mostly used for the characterization of the light scattering by surfaces and atmospheric aerosols. The majority of polarimetric measurements are compiled in the visible wavelength range, although near-IR data and even thermal infrared ones are getting available. Linear polarimetry is much more frequently applied compared to circular one, most likely since the former is found easier to measure and interpret. The current knowledge on solar system objects resulting from polarimetric observations is reflected in various review papers, for instance in Belskaya et al. (2008), Kolokolova et al. (2004), Muinonen et al. (2002).

The Stokes parameters for the complete description of the electromagnetic wave, i.e., $\mathrm{I}$ for the intensity, $\mathrm{Q}$ and $\mathrm{U}$ for linear polarimetry and $\mathrm{V}$ for circular polarimetry, are frequently normalized as $\mathrm{P}_{\mathrm{Q}}=\mathrm{Q} / \mathrm{I}, \mathrm{P}_{\mathrm{U}}=\mathrm{U} / \mathrm{I}$ and $\mathrm{P}_{\mathrm{V}}=\mathrm{V} / \mathrm{I}$. In planetary science one also calculates the so called linear polarization degree $\mathrm{P}$, i.e., the length of the linear polarization vector $\left(\mathrm{P}_{\mathrm{Q}}, \mathrm{P}_{\mathrm{U}}\right)$. In many observing applications of light scattering from planetary surfaces $\mathrm{P}$ only is determined, implicitly assuming $\mathrm{P}_{\mathrm{U}}=0$. The latter condition implies that the light is polarized linearly in or perpendicular to the light scattering plane Sunobject-observer, which is frequently the case, but better verified through independent measurements.

The FORS1 instrument (http://www.eso.org/sci/facilities/paranal/instruments/), a focal reducer and spectrograph at the Very Large Telescope VLT of the European Southern Observatory ESO at Cerro Paranal in Chile, represents a powerful polarimetric and spectropolarimetric facility at an $8 \mathrm{~m}$-class telescope and a first class site. Equipped with Wollaston prism, $\lambda / 2$ - and $\lambda / 4$-waveplate, FORS1 allows polarimetric measurements of solar system objects in the visible range as faint as $\mathrm{V} \sim 20 \mathrm{mag}$ and at high signal-tonoise ratio (500-1,000). Its application for planetary science and how to reduce and evaluate the FORS1 polarimetric data is described for instance in Boehnhardt et al. (2004) and Bagnulo et al. (2006, 2008). For very accurate measurements and for measurements of extended objects one has to consider special precaution in order to estimate the polarization field dependences and polarization cross-talk effects.

In the next section we summarize new polarimetric results achieved with FORS1 on solar system objects over the past few years. Based on this very positive experience we argue in Sect. 3 for polarimetric equipment to be available with instrumentation at the next generation of Extremely Large Telescope facilities (ELTs).

\section{Recent Polarimetry of Solar System Objects at the ESO VLT}

\subsection{Small Asteroids}

It has been demonstrated by Delbo et al. (2007) that measurements of the linear polarimetric phase function of the near-Earth object (99942) Apophis can provide high accuracy results on the albedo and-when combined with photometric data-on the size of minor asteroids. The accuracy of the results appears to be limited by the uncertainties in the calibration parameters of the empiric polarization-albedo relationship rather than by those of the measurements themselves. However, given the alternatives for albedo-size estimations (combined measurements of reflected and thermal spectrum of objects, radar 
reflections at the body, in situ imaging by space missions) it might be worthwhile to further explore the possibility of the polarimetric approach since this method appears reliable, accurate and 'cheap in terms of observing time,' and it can reach also the small population which is otherwise indeed difficult or expensive to accomplish.

\subsection{Kuiper Belt Objects}

In 2004 Boehnhardt et al. published the linear polarimetric phase function of the Plutino 28978 Ixion, the first ever Kuiper Belt object measured after Pluto. Restricted to the very narrow phase angle range of less than $1.5^{\circ}$ set by the large distance of the object, the available polarimetry and photometry allowed the conclusion on the presence of at least two surface components with rather different scattering properties. The next publication of this group of authors (Bagnulo et al. 2006) introduced similar results for the classical disk object 50000 Quaoar in the Kuiper Belt and for the transition object, the Centaur 2060 Chiron, the latter being another first representative of an interesting group of small solar system objects in the distance range beyond Jupiter. In the meanwhile, about a handful of Kuiper Belt objects (KBOs) were measured with FORS1 and first trends of polarimetric properties could be established (Bagnulo et al. 2008): It appears that the large KBOs display shallow polarimetric phase functions, while the smaller KBOs show steep negative polarization at small phase angles. Presently, there is no model interpretation available for this finding. It is however noteworthy to mention that the large size KBOs have also different surface chemistry from that of the smaller ones (Brown 2008), indicating resurfacing with ices that are not present in the other KBOs as such and may also affect the light scattering behavior.

\subsection{Cometary Nuclei}

Polarimetric measurements of cometary dust are presented well in literature (see, e.g., review by Kolokolova et al. 2004). Polarimetry of cometary nuclei is very sparse (Jewitt 2004). In 2008 Boehnhardt et al. published the first linear polarimetric phase function of the nucleus of comet $2 \mathrm{P} /$ Encke. An attempt to associate the measured function with that of other small solar system objects failed, indicating that at least $2 \mathrm{P} /$ Encke is unique in its polarimetric light scattering. Without polarimetry of other similar objects, it is however too early to conclude further on cometary nuclei per se. More recently, Bagnulo et al. (2009) presented the photometric and polarimetric phase functions of 133P/Elst-Pizarro, one of the four main belt comets known so far. The results put this object for its light scattering properties closer to F-type asteroids rather than to the nucleus of $2 \mathrm{P} /$ Encke. It remains however unclear whether to consider 133P/Elst-Pizarro as a cometary nucleus or an asteroid. It is worth mentioning that the polarimetric phase functions of both nuclei are distinctly different from those of cometary dust, despite the latter may be of the same material and may even have similar particle structure to that found on cometary surfaces.

\subsection{Cometary Coma Material}

The linear polarimetric phase function of coma material is measured for many comets (Kolokolova et al. 2004). One of the main findings in this field is the discovery of the dichotomy between the phase functions of gas-rich and gas-poor comets. It remains an open issue if this finding is real (Levasseur-Regourd et al. 1996) or maybe an artifact of 
different gas contamination of the measurements (Jockers et al. 2005) that are usually performed through filters of different bandwidths and at different central wavelength and of course at comets of different dust-to-gas ratios. Linear spectropolarimetry of comet 73P/ Schwassmann-Wachmann 3 using FORS1 at the VLT has revealed that the polarization degree drops considerably in the wavelength regions of cometary gas emission lines. This effect could be explained by the depolarizing signal of the possibly unpolarized (or only weakly polarized) coma gas. It should be noted that polarization of the gas component may be non-zero and measurable in case of gas fluorescence (Leborgne and Crovisier 1987) although so far no attempts were made to obtain the gas polarization from such spectropolarimetric data. Altogether, the split comet 73P/Schwassmann-Wachmann 3 falls in the category of dust-rich (or gas-poor) comets as already noted by Bonev et al. (2008). However, it was found that unlike for other dust-rich comets, the polarization degree of the dust continuum of comet SW3 did not depend on the wavelength (usually it increases with the wavelength for the wavelength range 400-900 nm).

The FORS1 observations provided also a first spectrum of circular polarimetry of this comet in the visible wavelength region. Within errors $(<0.2 \%)$ no circular polarization could be detected in SW3.

\subsection{Life Science}

It is well known that many molecules as building stones of terrestrial life (amino acids, sugars etc.) have chiral nature and that only one form of chirality is used in life forms on Earth. As a net result of its chiral nature, life forms (for instance plant leaves) display small circular polarization when reflecting (sun)light (Pospergelis 1969; Wolstencroft et al. 2002; Kolokolova and Sparks 2008). In order to benchmark future searches for (pre)biotic material in space and on the surface of other astronomical objects, it is important to verify the possibility to infer the existence of terrestrial life through circularly polarized light. The experiment was performed using FORS1 at the VLT and attempted to measure the reflectance spectrum of the Earthshine from the Moon, once when illuminated mostly by the Pacific Ocean region and once when Africa and its equatorial forest greens were in sight. The results of these observations (Sterzik and Bagnulo 2007) reached unprecedented small uncertainties. However, no firm detection of circularly polarized signatures were found, although a weak systematic structure of the $\mathrm{P}_{\mathrm{V}^{\prime}}$ polarization signal is noted around the spectral region of the Chlorophyll band around at 7,100 nm was found. A negative result was also found in the case of Mars when applying FORS1 imagery to search for circularly polarized light as a possible indicator for life on this planet (Sparks et al. 2005).

\section{Why Polarization Equipment at ELTs and What are the Requirements?}

\subsection{Polarimetry Science at ELTs}

The previous section has illustrated the cases of planetary science for polarimetric measurements: (1) Exploration of surface properties through light scattering reveals grain sizes, porosity, and albedo (without resolving the surface grains as such) and as a net result also the overall albedo of the surface. (2) Using spectropolarimetry in particular in the near-IR it should become feasible to explore such properties for individual surface materials. Cases (1) and (2) are of great interest for small asteroids, cometary nuclei, and KBOs as well as the whole 'zoo' of other small bodies, not even started to be explored by polarimetric 
measurements so far (irregular satellites of planets, Trojans). The existing telescope equipment allows in principle case studies of some individual objects, while survey-type systematic studies are virtually impossible because of the faintness of the objects and thus the lacking signal-to-noise for polarimetric measurements. Space missions will of course provide reference information on surface properties, however, only for very few objects (certainly less than a handful over the next two decades). (3) As for the surface exploration it will be possible to measure grain properties of cometary coma material after it has left the nuclear surface. It will be interesting to see whether more 'individuality'-beyond gasrich and 'gas-poor'-is found among comets. The polarization of the coma gas remains an open issue for the future. A rather unexplored area of research is the polarization of dust in planetary rings. (4) And last but not least and at present certainly of somewhat speculative nature: circular polarimetry may become a useful tool to search systematically for the existence of pre-biotic material in space through detectability of homo-chirality.

\subsection{Instrument Requirements}

Suitable polarimetric equipment at future ELT should allow imaging and spectropolarimetry (linear and circular) over a wide wavelength region from the UV to the mid-IR. A $40 \mathrm{~m}$-class telescope will automatically push the sensitivity limits by 3-4 mag compared to existing equipment at 8-10 m-class facilities, enabling sample and context studies of group properties that will otherwise never be done. It is certainly also required to allow highly accurate measurements at the level of $0.001-0.1 \%$ polarization, not only for the life science aspects of planetary research. The wide wavelength coverage is required at low dispersion (order 1,000) only. However, for the gas polarization detection one may benefit from high dispersion spectropolarimetry in the visible and in particular in the $3-5 \mu \mathrm{m}$ domain.

Open Access This article is distributed under the terms of the Creative Commons Attribution Noncommercial License which permits any noncommercial use, distribution, and reproduction in any medium, provided the original author(s) and source are credited.

\section{References}

S. Bagnulo, H. Boehnhardt, K. Muinonen, L. Kolokolova, I. Belskaya, M.A. Barucci, A\&A 450, 1239 (2006)

S. Bagnulo, I. Belskaya, K. Muinonen, G.P. Tozzi, M.A. Barucci, L. Kolokolova, S. Fornasier, A\&A 491, L33 (2008)

S. Bagnulo, H. Boehnhardt, G.P. Tozzi, J.-B.Vincent, A\&A, (2009) submitted

I.N. Belskaya, A.-C. Levasseur-Regourd, Y.G. Shkuratov, K. Muinonen, in The Solar System Beyond Neptune, ed. by M.A. Barucci, H. Boehnhardt, D. Cruikshank, A. Morbidelli (University of Arizona Press, Tucson, 2008), p. 115

H. Boehnhardt, S. Bagnulo, K. Muinonen, M.A. Barucci, L. Kolokolova, E. Dotto, G.P. Tozzi, A\&A 415, L21 (2004)

H. Boehnhardt, G.P. Tozzi, S. Bagnulo, K. Muinonen, A. Nathues, L. Kolokolova, A\&A 489, 1337 (2008)

T. Bonev, H. Boehnhardt, G. Borisov, A\&A 480, 277 (2008)

M.E. Brown, in The Solar System Beyond Neptune, ed. by M.A. Barucci, H. Boehnhardt, D. Cruikshank, A. Morbidelli (University of Arizona Press, Tucson, 2008), p. 335

M. Delbo, A. Cellino, E.F. Tedesco, Icarus 188, 266 (2007)

D. Jewitt, AJ 128, 3061 (2004)

K. Jockers, N. Kiselev, T. Bonev, V. Rosenbush, N. Shakhovskoy, S. Kolesnikov, Yu. Efimov, D. Shakhovskoy, K. Antonyuk, A\&A 441, 773 (2005)

L. Kolokolova, W. Sparks, AAS DPS 40, 40-02 (2008) 
L. Kolokolova, M.S. Hanner, A.-C. Levasseur-Regourd, B. Gustafson, in Comets II, ed. by M. Festou, H.U. Keller, H. Weaver (University of Arizona Press, Tucson, 2004), p. 577

J.F. Leborgne, J. Crovisier, ESA-SP 278, 171 (1987)

A.C. Levasseur-Regourd, E. Hadamcik, J.B. Renard, A\&A 313, 327 (1996)

K. Muinonen, J. Piironen, G. Shkuratov Yu, A. Ovcharenko, B.E. Clark, in Asteroids III, ed. by W.F. Bottke Jr, A. Cellino, P. Paolicchi, R.P. Binzel (University of Arizona Press, Tucson, 2002), p. 123

M.M. Pospergelis, Sov. Phys.-Astron. 12, 973 (1969)

W.B. Sparks, J.H. Hough, L.E. Bergeron, Astrobiology 5, 737 (2005)

M.F. Sterzik, S. Bagnulo, in Bioastronomy 2007, ASP Conference Series, eds. by D. Werthimer, K. Meech, J. Siefert, M. Mumma, 16-20 July 2007. (San Juan, Puerto Rico, 2007)

R.D. Wolstencroft, G.E. Tranter, D.D. Le Pevelen, in Bioastronomy 2002, IAU Symposium 213, eds. by R.P. Norris, F.H. Stootman. Astron. Soc. Pac., Ann Arbor (2002), p. 149 